Revue de l'Institut des langues et cultures

d'Europe, Amérique, Afrique, Asie et Australie

42 | 2021

Le politiquement correct : tabous, normes, transgressions

\title{
Le langage inclusif en français et en allemand : une tempête dans un verre d'eau?
}

Inclusive Language in French and German: A Storm in a Teacup?

Gendergerechte Sprache im Französischen und im Deutschen: ein Sturm im

Wasserglass?

Nathalie Schnitzer

\section{OpenEdition}

1 Journals

Édition électronique

URL : http://journals.openedition.org/ilcea/11623

DOI : 10.4000/ilcea. 11623

ISSN : 2101-0609

Éditeur

UGA Éditions/Université Grenoble Alpes

Édition imprimée

ISBN : $978-2-37747-251-2$

ISSN : 1639-6073

Référence électronique

Nathalie Schnitzer, «Le langage inclusif en français et en allemand: une tempête dans un verre d'eau? », ILCEA [En ligne], 42 | 2021, mis en ligne le 31 janvier 2021, consulté le 01 février 2021. URL: http://journals.openedition.org/ilcea/11623; DOI : https://doi.org/10.4000/ilcea.11623

Ce document a été généré automatiquement le 1 février 2021

(c) ILCEA 


\title{
Le langage inclusif en français et en allemand : une tempête dans un verre d'eau?
}

\author{
Inclusive Language in French and German: A Storm in a Teacup? \\ Gendergerechte Sprache im Französischen und im Deutschen: ein Sturm im \\ Wasserglass?
}

Nathalie Schnitzer

\section{Introduction}

1 Avant d'être une affaire de langage, la question de l'inclusion des femmes se pose dans l'espace public. En mars 2019, une étude de l'INA sur le taux d'expression des femmes dans les médias français a établi qu'elles « n'occupent en moyenne qu'un tiers du temps de parole à la télévision et à la radio ${ }^{1}$ ». Bien que ce taux ait augmenté au cours des dernières années, les femmes n'en restent pas moins sous-représentées. Moins audibles dans les médias en tant que sujets parlants, les femmes sont aussi moins visibles dans la langue en tant qu'objets de discours - et c'est cette inégalité, perçue par les féministes comme une forme de discrimination sexiste, que le langage inclusif tente de résorber : «L'ordre patriarcal a gravé un signe profond dans la forme linguistique, en délimitant et cernant les formes mêmes de la dénomination.» (Violi, 1987:32) La présente contribution, qui s'inscrit dans une perspective comparative, propose un état des lieux des initiatives pour la mise en œuvre d'un «langage non sexiste ${ }^{2}$ » en français et en allemand depuis la fin des années 1980.

2 En Allemagne où le langage inclusif s'applique dans de nombreux domaines de la vie publique, notamment dans les milieux universitaires, il ne serait pas politiquement correct de faire abstraction de ce nouvel usage. En France en revanche, l'opportunité d'aller au-delà de la seule féminisation des noms de personnes pour rendre la langue plus égalitaire n'est devenue un sujet de discussion dans les médias généralistes qu'à 
l'automne 2017, à l'occasion de la parution chez Hatier d'un manuel d'histoire rédigé selon le principe de l'écriture dite inclusive (Le Callennec \& François, 2017). Le débat national déclenché par cette publication montre à quel point le sujet divise, ce qui n'a rien d'étonnant dans un pays où la simple utilisation du féminin pour les noms de métiers, fonctions, grades et titres faisait encore polémique il y a peu. L'expression écriture inclusive est finalement entrée dans Le Robert en 2019. Le lexicographe et " père » du Robert, Alain Rey, ne lui prédisait pourtant pas un grand avenir. Dans une interview au Figaro, il déclarait au plus fort de la crise : «L'affaire de l'écriture inclusive est une tempête dans un verre d'eau. Dans six mois, plus personne n'en parlera ${ }^{3}$.»

On en parle encore aujourd'hui, en dépit de cette prédiction, mais il y a lieu de s'interroger sur l'avenir à plus long terme des pratiques inclusives. Ont-elles une chance de s'installer durablement dans l'usage et de rendre la langue moins androcentrée, tout en accompagnant les transformations de la société vers plus d'équité ? Ou bien cette entreprise politiquement correcte dans le mauvais sens du terme est-elle vouée à l'échec ? Il est sans doute trop tôt pour le dire et les observations qui suivent visent simplement à mettre en regard les faits de langue concernés et les stratégies proposées en français et en allemand en faveur d'un langage plus égalitaire.

4 Après avoir évoqué la manière dont le langage inclusif s'inscrit dans le mouvement politiquement correct, on reviendra sur les principales objections qu'il a suscitées, et suscite encore, y compris parmi les linguistes. L'accent sera mis ensuite sur les aspects techniques de sa mise en œuvre dans une langue et dans l'autre : féminisation des noms de personnes, processus d'épicénisation, remise en cause du masculin générique. On se demandera si la langue française, sa morphologie, sa grammaire, est plus réfractaire que l'allemand à l'application de mesures de rééquilibrage (voire de neutralisation) des genres, ce qui pourrait expliquer en partie le retard pris par la France dans ce domaine.

\section{Langage inclusif et politiquement correct}

5 La question est ici de savoir quel visage de la « tête de Janus » du politiquement correct (voir Schafroth, 2010) le langage inclusif nous donne à voir. Selon Schafroth, le langage inclusif relève, au moins en partie, de la force affirmative (die affirmative Kraft) du politiquement correct, qui vise à créer de nouvelles formes pour rendre linguistiquement visibles des réalités demeurées cachées jusqu'alors (voir Schafroth, 2010 : 101). Cette revendication n'est pas nouvelle et s'inscrit dans une logique d'émancipation qui remet en cause, non sans peine, l'ordre établi : «La création de formes de féminin pour les noms de métiers et la rébellion contre le masculin générique ont déclenché de véritables guerres de tranchées idéologiques dans la France des années 1980 et $1990^{4}$.» (Schafroth, $2010: 112$ ) Les revendications étaient pourtant modestes à l'époque : obtenir le droit d'user du féminin pour les fonctions de rang élevé, comme «madame la ministre » au lieu de «madame le ministre ». Le principe en avait été énoncé dès 1986 dans la circulaire Fabius, mais il fallut attendre celle du 6 mars 1998 pour qu'il soit réaffirmé et effectivement appliqué aux femmes du gouvernement Jospin, contre l'avis de l'Académie française.

6 Aujourd'hui, la lutte pour davantage de visibilité ne concerne plus seulement les femmes. La discussion a été récemment étendue aux personnes qui ne se reconnaissent pas dans la binarité des genres et revendiquent une identité propre, y compris dans la langue, grâce notamment à des néopronoms tels que iel ${ }^{5}$ pour la $3^{\mathrm{e}}$ personne $\mathrm{du}$ 
singulier en français (voir Alpheratz, 2019), ou son équivalent sier en allemand (voir Hornscheidt, 2012). La question est d'autant plus actuelle en Allemagne que la loi sur l'état civil a été modifiée en 2018 pour permettre aux enfants dont le sexe n'est pas clairement défini à la naissance d'être inscrit sur le registre des naissances sans cette indication, ou avec l'indication divers (PStG, $\$ 22^{6}$ ). Le texte précise également que les personnes adultes peuvent également faire modifier leur état civil a posteriori (\$ 45b).

7 Augmenter la visibilité des groupes jusque-là dominés (les femmes), ou ignorés (les personnes non binaires ${ }^{7}$ ), ne va pas sans réduire la visibilité des traditionnels dominants (les hommes). Il s'agit autrement dit de démasculiniser une langue jugée androcentrée. Cela consiste, comme on le verra dans la suite, à remplacer les tournures ressenties comme sexistes par d'autres plus acceptables socialement, voire à remettre en cause certaines règles grammaticales, ce qui situe également le langage inclusif $d u$ côté de la force négative (die negierende $\mathrm{Kraft}$ ) du politiquement correct, lequel vise "l'évitement de formulations qui - pour diverses raisons - ne sont pas (plus) opportunes pour désigner des notions ou des faits considérés comme délicats ou sensibles ${ }^{8}$ " (Schafroth, 2010: 102). Bien qu'elle ait suscité de vives polémiques, Schafroth ne considère pas la lutte pour la " féminisation lexicale » des années 1980 à la fin des années 2000 en France comme relevant de la face nuisible du politiquement correct, sans pour autant présumer de l'avenir :

Il reste cependant à espérer, et j'invoque ici une fois de plus le visage repoussant du politiquement correct, qu'aucune interdiction - de quelque nature que ce soit n'intervienne jamais dans cette question ${ }^{9}$ (Schafroth, $2010: 122$ ).

Pour Schafroth, la bascule du bon vers le mauvais politiquement correct se produit donc lorsque celui-ci engendre des "interdictions». Or lorsqu'une nouvelle manière de parler ou d'écrire cherche à s'imposer, il faut bien que le changement se fasse au détriment de l'ancien usage en vigueur, les adversaires de l'écriture inclusive l'ont bien compris.

\section{Objections contre le langage inclusif}

9 En France, la remise en cause à l'automne 2017 dans un manuel scolaire de la règle du masculin générique a provoqué un véritable tollé dans les médias. Le philosophe Raphaël Enthoven, pour qui «le désir d'égalité n'excuse pas le façonnage des consciences », y a vu " une agression de la syntaxe par l'égalitarisme », ou encore " une réécriture qui appauvrit le langage exactement comme le novlangue dans $1984^{10} »$. Le journal Marianne l'a assimilé à une forme de censure ${ }^{11}$, tandis que l'Académie française, à l'époque encore hostile à la féminisation des noms de métiers, lançait ce cri d'alarme : "Devant cette aberration "inclusive", la langue française se trouve désormais en péril mortel, ce dont notre nation est dès aujourd'hui comptable devant les générations futures ${ }^{12}$.»

Le langage inclusif est donc la cible de nombreuses critiques et les femmes elles-mêmes n'y sont pas toujours favorables. Il y a quelques années, Marina Yaguello expliquait déjà les réticences à la féminisation des titres de la part des principales intéressées comme relevant de «la phase phallique de la conquête du pouvoir où les femmes demandaient avant tout qu'on les traite comme des hommes » $(1998$ : 178). Pour Marie-Anne Paveau, il s'agissait plus prosaïquement d'un «bel exemple d'intériorisation des axiologies dominantes» $(2002: 124)$. Aujourd'hui encore, dans un registre plus linguistique, on 
entend dire que ce n'est pas la langue qui est sexiste, mais les locuteurs... et les locutrices qui l'utilisent. Si la langue n'a aucune influence sur les mentalités, et vice versa, le langage inclusif relève effectivement du politiquement correct dans son acception négative, puisqu'il revient à dissimuler les inégalités sociales persistantes entre hommes et femmes derrière une égalité linguistique de façade. À cela on peut objecter que l'accusation portée par les mêmes adversaires du langage inclusif contre ce "novlangue ", coupable d'appauvrir la pensée par le langage, est pour le moins contradictoire (voir Abbou, Arnold, Candea \& Marignier, 2018: § 23 [en ligne]). De deux choses l'une : soit la langue est un outil sociologiquement et politiquement inerte et aucun novlangue ne peut constituer une menace pour la pensée, soit la langue est en interaction avec les réalités sociales et politiques, et alors on peut s'attendre à ce que le fait de la transformer affecte les mentalités. La question est alors de savoir quelles transformations sont politiquement acceptables, voire souhaitables.

11 Un argument linguistique plus technique contre l'adoption d'un langage inclusif consiste à rappeler inlassablement que sexe et genre ne doivent pas être confondus, et que la règle du masculin générique ne signifie pas que le masculin l'emporte sur le féminin, mais qu'il neutralise l'opposition masculin-féminin. Du côté allemand, cette thèse est notamment défendue par Eisenberg, qui s'est beaucoup engagé sur le sujet ces dernières années (voir Eisenberg, 2017). Du côté français, ses arguments rejoignent ceux de Charaudeau (2018 et 2019), pour qui le masculin générique est un processus grammatical de neutralisation qui subsume les deux genres dans une catégorie englobante :

[...] dans « les 800000 lycéens et étudiants en réorientation commencent à émettre leurs vœux d'orientation ", le générique collectif joue à plein son rôle, et on ne voit pas pourquoi le sexe féminin serait exclu. (Charaudeau, 2019:118)

Cette présentation du genre dans la langue comme une réalité purement grammaticale est vivement contestée par les linguistes pour qui la langue est aussi une réalité sociale :

Bien que la grammaire soit a priori indifférente à l'idéologie, l'usage subit l'influence des mentalités et des représentations sociales. S'agissant des référents humains, le genre grammatical n'est pas tout à fait innocent en ce qu'il contribue à la fabrication du « genre social ». (Yaguello, 2014 : 11-12)

13 Parmi les arguments linguistiques avancés contre l'écriture inclusive, il faut encore évoquer celui de la dangerosité que représenterait une telle atteinte au système de la langue. Cette inquiétude est notamment exprimée pour l'allemand par Leiss, qui recommande de «ne jamais interférer dans un système dont tu ne perçois pas toute la complexité ${ }^{13}$ "; le langage inclusif entraîne selon Leiss une extrême dramatisation de l'appartenance sexuelle ayant pour effet pervers de renforcer les clivages au lieu de les résorber. Cet argument de la sexualisation de la langue constitue bien un point délicat. En effet, plus le langage inclusif gagne du terrain, moins le masculin dit générique peut encore être ressenti comme neutre : «À partir du moment où l'on a une étudiante à côté d'un étudiant, une fonctionnaire à côté d'un fonctionnaire, une cliente à côté d'un client, le générique masculin perd son caractère générique et est associé à un nom masculin ${ }^{14}$.» (Schneider-Mizony, 2007:123) Mais pour Schneider-Mizony, la responsabilité historique de la sexualisation de la grammaire n'est pas du côté des femmes, qui revendiquent dorénavant plus de visibilité dans la langue, mais du côté des grammairiens qui s'étaient auparavant attachés à promouvoir le masculin générique au détriment de la référence au féminin (voir Schneider-Mizony, 2007 : 124). 


\section{Féminisation des noms} langage inclusif commence avec l'usage du masculin et du féminin pour désigner les personnes. Contrairement aux noms d'objets et à la plupart des noms d'animaux, dont le genre est généralement arbitraire en synchronie, il existe ici une corrélation entre genre naturel (sexus) et genre grammatical (genus). Le sexe biologique des personnes se manifeste dans le groupe nominal par le biais :

- de bases nominales spécifiques [+fém] ou [+masc]: femme/homme, Frau/Mann, fille/fils, Tochter/Sohn...

- de bases nominales suffixées (morphèmes de flexion ou de dérivation ${ }^{15}$ ): boulanger/ boulangère, Bäcker/Bäckerin, acteur/actrice, Schauspieler/Schauspielerin...

- d'éléments associés à cette base (déterminants, adjectifs) : la/le, die/der, cette/ce, diese/dieser, belle/beau, schön/schöne...

- d'éléments se substituant au groupe (pronoms) : elle/il, sie/er, chacune/chacun, jede/jeder, celle qui/celui qui, diejenige die/derjenige der...

En allemand, où coexistent trois genres grammaticaux, le neutre ne désigne que rarement des êtres humains. C'est le cas pour das Mädchen/la jeune fille ou das Fräulein/ la demoiselle pour des raisons morphologiques (présence du suffixe diminutif -chen ou lein), ou encore pour das Baby/le bébé ou das Kind/l'enfant pour des raisons sémantiques. Mais les exemples se comptent sur les doigts de la main. Le fait de posséder un genre grammatical neutre ne rend donc pas nécessairement plus facile l'adoption d'un langage inclusif en allemand, le neutre désignant la plupart du temps des objets. En allemand comme en français, le masculin est utilisé non seulement dans son sens spécifique, mais aussi dans un sens générique, comme dans ce passage de la Loi fondamentale de la République fédérale (l'équivalent de la Constitution en France) :

Art. 2 - (1) Jeder hat das Recht auf die freie Entfaltung seiner Persönlichkeit, soweit er nicht die Rechte anderer verletzt und nicht gegen die verfassungsmäßige Ordnung oder das Sittengesetz verstößt.

Art. 2 - (1) Chacun a droit au libre épanouissement de sa personnalité pourvu qu'il ne viole pas les droits d'autrui ni n'enfreigne l'ordre constitutionnel ou la loi morale ${ }^{16}$.

\subsection{Noms de personnes en français}

Il existe différentes manières de former des féminins en français. La plus courante est l'ajout d'un -e final, associé ou non à une autre modification (boulangère, gardienne, sportive, voisine). Les autres options vont de l'absence de marque spécifique pour les épicènes (architecte), à l'ajout de -esse (hôtesse, maîtresse), en passant par le remplacement des finales en -eur par-euse (coiffeuse, conteuse), -eure (supérieure), ou -rice (ambassadrice, institutrice). Bien que le français ne manque pas de ressources pour rendre visible ce qu'il faut bien appeler le " deuxième genre " (le féminin grammatical étant toujours perçu comme second par rapport au masculin), la féminisation est encore hésitante dans certains cas, notamment lorsqu'il s'agit de noms de métiers, grades et fonctions autrefois réservés aux hommes : madame le président directeur général ou madame la présidente directrice générale? 
17 En français, le non-respect de la règle d'accord en genre a été justifié à maintes reprises par l'Académie au nom de la neutralité des fonctions, titres et grades, mais n'explique en rien la différence de traitement entre la "directrice d'école» ou la "maitresse d'école » d'une part, et le «directeur général » ou le «maitre de conférences » d'autre part. Les choses évoluent néanmoins et même l'Académie a fini par changer de position sur le sujet ${ }^{17}$. À l'université, de plus en plus de femmes se désignent volontiers comme maitresses de conférences, ce qui est assez récent. L'oreille française s'est également habituée à certaines formes de féminin autrefois aussi infréquentables que leurs référentes : en 1992, le Petit Robert définit uniquement le terme entraîneuse comme une « jeune femme employée dans les bars pour entraîner les clients à danser (cf. Taxigirl), à consommer "; dans l'édition de l'an 2000 du même dictionnaire, on peut lire pour illustrer la définition du métier d'entraîneur sportif : "l'entraîneuse de notre championne ", signe que ce féminin est entré dans les (bonnes) mœurs linguistiques. Une telle évolution donne raison à Paveau pour qui l'argument des connotations sexuelles associées à certains mots féminisés (elle cite également: coureuse, tôlière, monteuse, sauteuse) est peu valable «car les connotations sont évidemment de nature sociale et culturelle, et non pas données par la langue [...]» (2002:124).

Même en dehors de toute référence sexuelle, l'homonymie est encore considérée comme un obstacle à la féminisation, bien que le phénomène existe déjà et ne semble guère poser problème : avocat (le juriste ou le fruit), hérö̈ne (féminin de héros ou drogue dure). La fréquence de l'homonymie constitue certes une difficulté pour l'apprentissage du français langue étrangère, mais n'est pas en soi considérée comme un défaut de la langue. Pour cuisinière (femme qui cuisine) et cuisinière (poste de cuisson), la question se pose uniquement dans le milieu de la haute cuisine, comme on peut le vérifier en visitant les pages Wikipédia de professionnelles concernées :

Virginie Basselot, née le 21 avril 1979 à Deauville, est une femme chef cuisinière française, Meilleur ouvrier de France ${ }^{18}$.

Anne-Sophie Pic, née le 12 juillet 1969 à Valence (Drôme), est un chef cuisinier et maître restaurateur ${ }^{19}$.

Stéphanie Le Quellec, née Lecocq le 6 décembre 1981 à Enghien-les-Bains (Vald'Oise) est une femme chef cuisinier française ${ }^{20}$.

19 En comparaison, les pages Wikipedia des cuisinières étoilées allemandes Julia Komp ${ }^{21}$, Meike Menzel $^{22}$ et Douce Steiner ${ }^{23}$ déçoivent presque par leur uniformité. Les articles qui leur sont consacrés s'ouvrent invariablement sur la formule " [...] ist eine deutsche Köchin " (" est une cuisinière allemande »). L'excellence ne semble pas ici constituer un obstacle à la féminisation du nom de métier.

\subsection{Noms de personnes en allemand}

En allemand, la féminisation lexicale (Movierung, Motion) n'est pas un sujet polémique. Une entraîneuse sportive est eine Trainerin (masc. Trainer), une femme médecin eine Ärztin (masc. Arzt). La plupart des noms de personnes, et notamment de métiers, forment leur féminin en -in ${ }^{24}$ (Bäckerin, Maurerin, Schriftstellerin - boulangère, maçonne, écrivaine), sans entraîner de connotations indésirables (voir Bußmann \& Hellinger, 2003 : 167). L'idée que certains nouveaux termes (Doktorin, Professorin) à la sonorité inhabituelle doivent être patinés par l'usage pour que leur emploi devienne naturel est généralement bien acceptée. Pour ce qui est de Kanzlerin, le succès fulgurant de ce féminin est évidemment lié à l'arrivée d'Angela Merkel au poste de chancelière en 2005. 
S'ils avaient suivi les recommandations de l'Académie, les médias français auraient dû à l'époque employer la formule "madame le chancelier Merkel » pour désigner cette cheffe de gouvernement; mais le guide Femme j'écris ton nom (INaLF, 1999), rédigé à l'initiative du gouvernement Jospin, avait manifestement déjà fait son chemin dans les rédactions ${ }^{25}$.

Contrairement au français, où même lorsque la forme de féminin est attestée, son emploi n'est pas nécessairement admis dans tous les contextes, il existe assez peu de cas difficiles en allemand, les masculins en -eur (féminin en -euse/-öse) et en -ling (sans féminin) faisant exception. Les féminins de noms d'emprunt ayant un suffixe en -euse (öse) peuvent être connotés négativement, ce qui explique la régression des emplois de Friseuse/Frisöse (la coiffeuse) et la progression du féminin en -in correspondant : Friseurin. Selon le Duden, «le terme Friseuse n'est plus employé qu'en langue familière; le terme officiel est Friseurin ${ }^{26} »$. Cette tendance est confirmée par la courbe d'évolution de ces mots dans la base de données du DWDS ${ }^{27}$. Autre exemple de connotation péjorative associée au suffixe -euse : die Masseuse vs die Masseurin (voir Elmiger, 2008 : 103). Selon le Duden, «le terme correct pour désigner le nom de métier [consistant à pratiquer des massages] est Masseurin; le terme Masseuse est essentiellement un euphémisme pour parler d'une prostituée ${ }^{28} »$.

Les nombreux mots masculins formés avec le suffixe -ling n'ont pas de forme de féminin ${ }^{29}$, sans avoir pour autant le statut de véritables épicènes. Pour les noms de personnes, la solution consiste à trouver des formes alternatives. C'est ainsi que Lehrling (l'apprenti, plutôt de sexe masculin) tend à être remplacé par die/der Auszubildende (la [personne] à former) qui a l'avantage de désigner indifféremment un ou une apprenti.e. Pour ce qui est du terme Flüchtling (le réfugié), il est concurrencé par les participes 1 et 2 substantivés du verbe flüchten (fuir) qui sont l'un comme l'autre épicènes : die/der Flüchtende (la [personne] qui fuit), die/der Geflüchtete (la [personne] qui a fui). Les adversaires de la féminisation objectent cependant que Flüchtling, Flüchtender et Geflüchteter ont des sens différents (voir Eisenberg, $2017: 69$ ).

\section{Mots épicènes, alliés de l'inclusion}

Les épicènes sont des mots (noms, adjectifs, pronoms) «qui ont la même forme au masculin et au féminin" $\left(T L F^{30}\right)^{30}$. La plupart des noms épicènes du français ont une finale en -e et peuvent être à genre fixe, tantôt masculin (un membre), tantôt féminin (une personne), ou à genre variable (un/une élève). Le mot maire, traditionnellement de genre masculin (madame le maire), autrefois féminisé à l'aide du suffixe -esse (madame la mairesse $e^{31}$ ), a finalement rejoint le groupe des épicènes à genre variable (madame la maire). Ce phénomène de dédoublement du genre est actuellement assez productif, comme on le constate aussi avec la féminisation de témoin dans les médias (toujours masculin dans les dictionnaires) :

DÉFI : 7 jours pour être la témoin idéale

Vous pensiez être demoiselle d'honneur, vous voilà promue témoin. Une mission de taille, à prendre au sérieux. (Le Parisien, 22 avril 2018)

Le masculin ${ }^{32}$ membre, dont on trouve aujourd'hui des occurrences au féminin, suit la même tendance :

La France décore une membre du gouvernement Orbán de la légion d'honneur.

(Mediapart, 31 janvier 2019) 
dédoublement du genre, comme: artiste, cadre, élève, guide, interprète, partenaire, locataire, propriétaire, scientifique, secrétaire... Autant de termes strictement masculins en allemand avec leur finale en -er (ou -är) : Künstler, Kader, Schüler, Führer, Dolmetscher, Partner, Mieter, Besitzer, Wissenschaftler, Sekretär. Enfin, le français possède pour qualifier les personnes nombre d'adjectifs épicènes que les guides allemands de langage inclusif pourraient nous envier : aimable, brave, jeune, honnête, paisible, pauvre, riche, tranquille... En allemand, les formes de singulier des adjectifs freundlich, mutig, jung, ehrlich, ruhig, arm, reich, ruhig, etc. se déclinent de manière distincte au masculin et au féminin (la fameuse déclinaison dite forte de l'adjectif).

L'expression allemande consacrée pour désigner les épicènes est geschlechtsneutrale Bezeichnungen (désignations neutres en genre), ce qui ne signifie pas que les noms concernés soient de genre grammatical neutre, comme on pourrait l'attendre d'une langue qui possède cette catégorie grammaticale. Parmi les épicènes à genre fixe ${ }^{33}$, le féminin du mot Person s'explique par son origine latine. Le masculin Star, emprunté à l'anglais, est proche de der Stern (l'étoile). Un autre masculin, der Mensch (l'humain), présent par exemple dans le composé Menschenrechte, permet aux germanophones de faire l'économie de la discussion sur les droits de l'homme ou les droits humains. Parmi les classiques, attestés aussi en français, figurent également des neutres : das Mitglied, das Opfer, das Personal (le membre, la victime, le personnel), ainsi que le pluriel die Leute (les gens). Mais la majorité des épicènes allemands sont des adjectifs et participes substantivés: die/der... Abgeordnete, Angestellte, Reisende, Arbeitslose, Kranke, Fremde, Angehörige, Deutsche (la/le/l'... député.e, employé.e, voyageuse/voyageur, chômeuse/chômeur, malade, proche, Allemand.e). Ce modèle est si inclusif qu'il a été utilisé ces dernières années pour créer de nouveaux épicènes en remplacement de termes strictement masculins (comme on l'a vu pour Flüchtling > Flüchtender/Geflüchteter). Au défini singulier, ces néologismes évitent d'avoir recours au dédoublement du nom (mais pas de l'article) :

- der Student (l'étudiant) > die/der Studierende (l'étudiant.e);

- der Lehrer (le professeur) > die/der Lehrende (l'enseignant.e);

- der Teilnehmer (le participant) > die/der Teilnehmende (la/le participant.e).

Au pluriel, les groupes nominaux ayant pour base ces participes 1 substantivés sont totalement épicènes (l'opposition de genre n'étant pas marquée par les éléments associés à la base nominale, articles et autres déterminants éventuels), ce qui explique que la neutralisation par le pluriel fasse aussi partie des stratégies recommandées par les guides allemands de langage inclusif comme alternative au splitting: die... Studierenden, Lehrenden, Teilnehmenden (les... étudiant.es, enseignant.es, participant.es). Cette nouvelle stratégie entre en concurrence avec la convention déjà ancienne du Binnen-I (I médian) : le -i du suffixe féminin -in noté en majuscule indique qu'un terme féminin est employé au sens générique: die LehrerInnen, die StudentInnen, die TeilnehmerInnen.

En français, selon les règles en vigueur, les participes et adjectifs substantivés ont également le genre grammatical correspondant au sexe des personnes auxquelles ils renvoient : une assistante sociale, un employé consciencieux, une prostituée mineure, un jeune retraité. S'agissant des fonctions socialement valorisées, l'accord en genre des participes et adjectifs substantivés a pourtant longtemps fait débat au mépris de la règle grammaticale, en grande partie du fait de la position conservatrice de l'Académie 
française : le principal de collège, l'attaché culturel, le chargé de mission. Autre inconvénient majeur: les équivalents français de die/der Studierende, die/der Lehrende, die/der Teilnehmende ne sont épicènes ni au singulier ni au pluriel (les marques de genre étant toujours maintenues) : l'étudiant vs l'étudiante, les étudiants vs les étudiantes.

L'allemand fait en outre volontiers appel à des composés dont la base permet de neutraliser l'opposition masculin/féminin, tels que :

- Kraft : die Lehrkraft / l'enseignant.e;

- Person : die Ansprechperson / la personne à contacter ;

- Hilfe : die Bibliothekshilfe / l'auxiliaire de bibliothèque.

Paradoxalement, la neutralisation n'est pas systématiquement souhaitée : certes utile pour les emplois génériques, elle peut aussi avoir pour effet indésirable de faire perdurer l'invisibilité des femmes dans les emplois spécifiques, ce qui explique la création en allemand de formes de féminin pour les titres notés en abrégé (la désinence -in du féminin est ajoutée en exposant): Professorin: Prof. ${ }^{\text {in }}$, Doktorin: Dr. ${ }^{\text {in }}$, DiplomDesignerin: Dipl.-Des. ${ }^{i n}$, peut-on lire dans le guide de langage inclusif des universités du Land de Brême $\left(2014: 11^{34}\right)$.

\section{Masculin générique en voie d'exclusion?}

La règle du masculin générique intervient au niveau lexical en imposant l'utilisation du substantif masculin pour désigner une ou des personnes en général, sans distinction de sexe : le mot écrivain/Schriftsteller peut référer aussi bien à un homme qu'à une femme. Mais cette règle a également des implications phrastiques, voire transphrastiques. En effet, le phénomène de l'accord " peut se décrire comme une contrainte exercée par un élément sur la forme d'un ou plusieurs autres éléments du syntagme ou de la phrase où il figure, et parfois même au-delà » (Riegel, Pellat \& Rioul, $1994: 897$ ). Se pose alors la question du comportement des "autres éléments" soumis à l'accord, notamment celui de l'adjectif.

Ici l'allemand possède incontestablement un double avantage: pour l'adjectif en position d'épithète, la distinction entre masculin et féminin n'est marquée qu'au singulier (et à certaines formes) ; pour l'adjectif en position d'attribut, le problème ne se pose pas puisqu'il est invariable, ce qui est aussi le cas du participe 2 :

- Les petits enfants / die kleinen Kinder;

- Elvira et Léonard sont partis / Elvira und Léonard sind gegangen;

- César et Rosalie sont amoureux / César und Rosalie sind verliebt.

Le cas des pronoms personnels est assez intéressant: en français, le pronom sujet de troisième personne du pluriel est marqué en genre (elles/ils disent); en allemand, le pronom pluriel sie (sie sagen) est perçu comme épicène même si, comme le fait remarquer Meinunger, sa forme est identique à la forme de féminin singulier (sie/elle), ce qui est également vrai de l'article défini pluriel (die/les), identique à la forme de l'article défini féminin singulier (die/la). La langue allemande ne serait donc, selon lui, pas si sexiste qu'on le prétend, l'allemand possèderait même "un féminin générique totalement ignoré » (Meinunger, $2017: 94^{35}$ ). Cette supposée prédominance du féminin au pluriel compenserait la prédominance du masculin au singulier, ce qui serait la preuve d'un équilibre dans la langue entre le masculin et le féminin (voir Meinunger, $\left.2017: 96^{36}\right)$. On peut objecter que cette impression de féminin ne change rien au fait que 
la règle du masculin générique s'applique bel et bien au substantif, en dépit de la forme superficiellement féminine de l'article pluriel (die) :

- Die Teilnehmer der Konferenz... / les participants du colloque...

- Die Antragsteller müssen das Formular vollständig ausfüllen / Les candidats doivent renseigner intégralement le formulaire.

Pomme de discorde par excellence, la règle du masculin générique est contestée depuis longtemps au motif que «le caractère prétendument générique du masculin est éclipsé par sa référence spécifique au sexe masculin et [qu']il est toujours plus difficile à utiliser lorsqu'il est censé identifier une femme en tant que telle » (Schneider-Mizony, $2007: 119^{37}$ ). Pour éviter de devoir continuer à enseigner aux élèves que le masculin l'emporte toujours sur le féminin, une solution envisageable pour le français serait le recours à l'accord de proximité, ou de voisinage, qui consiste à accorder le receveur (p. ex. l'adjectif ou le participe) avec le donneur le plus proche (p. ex. le nom) ${ }^{38}$ et, dans certains contextes, le recours à l'accord de majorité :

- Léonard et Elvira sont parties ;

-4 filles et 2 garçons sont admises à l'examen.

Mais la partie n'est pas gagnée pour les Modernes (notamment : Abbou, Arnold, Candea \& Marignier, 2018), car changer une règle de grammaire, même si elle n'est pas aussi canonique qu'on le croit, ne peut manquer d'irriter les Anciens (voir par exemple: Charaudeau, 2018 et 2019). Tout cela pourrait donner à penser que le français cumule les handicaps alors que l'adoption d'un langage rigoureusement inclusif en allemand nécessiterait beaucoup moins de bouleversements. Ce n'est pas tout à fait exact comme on va le voir à présent à partir de deux exemples particulièrement retors - parce qu'ils concernent des éléments du lexique quasiment incontournables dans la langue courante: man, l'équivalent de notre pronom indéfini on, et sein l'équivalent du possessif $s o n / s a$ [+ possesseur masculin spécifique ou générique].

\section{Man et sein font de la résistance !}

Homophone et quasi homographe du substantif Mann (homme), dont il est issu étymologiquement, le pronom indéfini sujet man est perçu comme potentiellement discriminant et les guides de langage inclusif recommandent d'éviter autant que possible son emploi ${ }^{39}$. Par exemple en recourant à une forme de passif ou en transposant la phrase à une autre personne :

- Man muss das Formular vollständig ausfüllen / On doit renseigner intégralement

le formulaire.

> Das Formular ist vollständig auszufüllen / Le formulaire est à renseigner intégralement.

- Man sollte sich mehr Gedanken darüber machen / On devrait davantage y réfléchir.

> Wir sollten uns mehr Gedanken darüber machen / Nous devrions davantage y réfléchir.

Selon Meinunger (2017 : 99), les graphies différentes de man et Mann indiquent assez clairement l'absence de lien sémantique entre les deux unités. Il rappelle également que deux autres pronoms extrêmement courants ont également été formés sur Mann: jemand (quelqu'un) et niemand (personne), mais le lien étymologique n'étant plus aussi perceptible (grâce à l'ajout $d u-d$ final), cette étymologie bien encombrante passe généralement inaperçue (Meinunger, $2017: 100$ ). Il n'en reste pas moins que le pronom man est devenu bien indésirable et que tous les moyens sont bons pour contourner son 
emploi - jusqu'à le remplacer par le néologisme frau (pronom dérivé du substantif Frau/femme), lorsque le contexte s'y prête :

- Über die neue Abtreibungsregelung muss frau sich genau informieren ${ }^{40} /$ Femme

[pronom !] doit s'informer précisément sur la nouvelle législation en matière d'avortement.

Ce nouveau pronom, utilisé depuis les années 1990 dans les publications féministes, est également attesté dans les médias généralistes. S'il n'est pas encore totalement grammaticalisé selon la grammaire de l'IDS (Institut für Deutsche Sprache ${ }^{41}$ ), son emploi n'en est pas moins suggéré dans plusieurs guides allemands de langage inclusif $^{42}$.

L'équivalent français du pronom man (on), qui est issu étymologiquement du substantif homme, ne fait l'objet d'aucune critique du même ordre. Deux raisons possibles à cela : d'abord on et homme ne sont pas homophones et encore moins homographes (l'étymologie commune ne saute donc ni aux yeux ni aux oreilles); ensuite homme est traditionnellement réputé désigner aussi bien l'être humain de sexe masculin que l'être humain en général - ce qui n'est pas le cas de Mann (l'allemand possédant un terme générique pour désigner l'être humain : Mensch).

Un autre casse-tête de la rédaction inclusive en allemand est le déterminant possessif sein. La forme de ce possessif de troisième personne du singulier est en effet différente selon qu'on a affaire à un possesseur (sein-) ou à une possesseuse (ihr-), comme c'est aussi le cas en anglais (her/his), alors qu'en français le sexe de la personne ne joue aucun rôle :

- La guitare de Léa > sa guitare : ihre Gitarre;

- La guitare de Léo > sa guitare : seine Gitarre.

41 Au masculin générique, c'est donc logiquement la forme sein qui est employée pour marquer le possessif, comme dans ce passage de la Loi fondamentale :

Art. 3 - (3) Niemand darf wegen seines Geschlechtes, seiner Abstammung, seiner Rasse, seiner Sprache, seiner Heimat und Herkunft, seines Glaubens, seiner religiösen oder politischen Anschauungen benachteiligt oder bevorzugt werden. Niemand darf wegen seiner Behinderung benachteiligt werden.

Art. 3 - (3) Nul ne doit être discriminé ni privilégié en raison de son sexe, de son ascendance, de sa race, de sa langue, de sa patrie et de son origine, de sa croyance, de ses opinions religieuses ou politiques. Nul ne doit être discriminé en raison de son handicap ${ }^{43}$.

Il existe depuis 2012 une version révisée de la Loi fondamentale en langage inclusif ${ }^{44}$. Dans l'article cité ci-dessus, la formule wegen seines Geschlechts, seiner Abstammung, etc. (en raison de son sexe, de son origine, etc.) a été remplacée par aufgrund von Geschlecht, von Abstammung, etc. (pour raison de sexe, d'origine, etc.). Mais ce texte n'a jusqu'à présent pas franchi le seuil des cercles militants.

Les deux « résistants » man et sein montrent que même si l'allemand possède certains avantages sur le français en matière de neutralisation (absence d'accord de l'adjectif attribut et du participe 2, effacement des marques de genre dans le GN pluriel), d'autres représentants du masculin générique contribuent à entraver la mise en œuvre d'une langue égalitaire. En adoptant (à la surprise générale) la règle du féminin générique dans son règlement intérieur de $2013^{45}$, l'université de Leipzig a agi avec pragmatisme. Cette mesure, qui consiste purement et simplement à retourner la logique du masculin générique, était en effet techniquement plus facile à appliquer que toute autre convention inclusive: "Dans ce règlement, les termes grammaticalement féminins 
pour désigner les personnes s'appliquent aux personnes de sexe masculin comme féminin ${ }^{46}$. "

Les médias d'outre-Rhin ont accueilli par un concert de moqueries ce renversement d'une norme linguistique perçue par une partie de la communauté linguistique comme immuable. Anatol Stefanowitsch, défenseur de l'application à la langue de la Règle d'or ( «ne représente pas les autres linguistiquement comme tu n'aimerais pas qu'on te représente à leur place ${ }^{47} »$ ), considère la lecture de ce document comme un moyen pour les hommes de prendre conscience de ce que signifie le fameux Mitgemeintsein (littéralement le fait d'être pensé avec - c'est-à-dire exister dans l'implicite) que vivent les femmes au quotidien; une expérience qu'il juge «légèrement déroutante et par conséquent très instructive ${ }^{48}$ ».

\section{Pour conclure}

Ce tour d'horizon des faits de langue concernés par la mise en œuvre du langage inclusif a permis de souligner des points de convergence et de divergence entre les deux langues. Au chapitre de la féminisation des noms de personnes dans les emplois spécifiques, les obstacles d'ordre morphologique et sémantique (homonymies, connotations indésirables) semblent plus nombreux côté français, mais ils ne sont pas insolubles - les formes féminines posent surtout un problème d'acceptabilité, qui se résorbe au fur et à mesure qu'elles entrent dans l'usage (ingénieure, maitresse de conférences, grande cuisinière). Concernant les épicènes, le français paraît en revanche plutôt mieux loti, avec ses nombreux noms de personne à finale en -e, à genre fixe ou variable (élève, maire, membre). L'allemand a de son côté l'avantage de puiser dans un stock important d'adjectifs et participes substantivés partiellement épicènes au singulier et totalement au pluriel (die Deutschen), et de pouvoir en créer de nouveaux en remplacement de noms marqués en genre (die Studierenden au lieu de die Studenten). À l'échelle de la phrase, le français a la particularité d'accorder ses adjectifs attributs et ses participes passés (invariables en allemand), un phénomène qui ne facilite pas la neutralisation des discours. La réactivation de l'accord de proximité (voire de l'accord de majorité) en remplacement du masculin générique pourrait venir compenser partiellement ce handicap. En allemand, certains déterminants et pronoms aussi incontournables qu'explicitement masculins donnent du fil à retordre aux meilleures volontés engagées dans la lutte contre l'androcentrisme. Le possessif sein vs ihr, dont la forme dépend du genre de la personne qui "possède ", constitue une réelle difficulté comme on l'a vu avec l'exemple emprunté à la Loi fondamentale. Quant à la discussion autour du pronom indéfini man, elle n'a pas non plus d'équivalent en français. Et il aurait encore fallu évoquer l'épineux problème des pronoms relatifs der et wer (voir Pittner, 1998), l'un et l'autre masculins, ce qui n'a pas été possible faute de place.

En allemand comme en français, l'accord au masculin générique est bien ce que la langue a produit de moins politiquement correct, même si l'étendue et les modalités d'application de cette règle divergent de part et d'autre. En allemand, la solution radicale consistant à remplacer le masculin générique par un féminin générique, mise en œuvre par l'université de Leipzig dans son règlement intérieur de 2013, n'a jusqu'à présent pas fait école. Si le masculin générique vacille sous les coups de boutoir du langage inclusif, son remplacement par un système plus équitable est loin d'être acté. De nouvelles propositions continuent d'émerger, entrant parfois en concurrence avec 
des conventions qui semblaient entrer progressivement dans l'usage (die Studierenden au lieu de die StudentInnen par exemple). Et la remise en cause de la binarité des genres, qui fait exploser la dichotomie ancestrale masculin/féminin, promet encore bien des débats. Les adversaires du langage inclusif peuvent y voir le signe qu'une telle entreprise est vouée à l'échec. À moins que cette période de grande effervescence ne soit une occasion d'explorer les potentialités de la langue, dans l'espoir de parvenir un jour à des solutions pérennes - et politiquement correctes au sens inclusif du terme, afin que le langage ne représente plus pour personne « une exclusion et une négation, le lieu où la structure patriarcale est ratifiée et inscrite »(Violi, 1987 : 32).

\section{BIBLIOGRAPHIE}

ABbou Julie, ARnold Aron, CANDEA Maria \& MARIGNIER Noémie (2018), «Qui a peur de l'écriture inclusive ? Entre délire eschatologique et peur d'émasculation. Entretien », Semen, 44, <https:// doi.org/10.4000/semen.10800>.

AlPHERATZ My (2019), « Français inclusif : du discours à la langue ?», Le discours et la langue, 11(1), 53-74.

BußMANN Hadumod \& HELLINGER Marlis (2003), « Engendering Female Visibility in German », M. Hellinger \& H. Bußmann (dir.), Gender Across Languages, Amsterdam / Philadelphie :

J. Benjamins, 3, 141-174.

CHARAUDEAu Patrick (2018), «L'écriture inclusive au défi de la neutralisation en français », Le Débat, 199(2), 13-31.

CHARAUDEAU Patrick (2019) : « Retour sur l'écriture inclusive au défi de la neutralisation en français ", Le discours et la langue, 11(1), 97-124.

CheValier Yannick, Constantin De Chanay Hugues \& Gardelle Laure (2017), « Bases linguistiques de l'émancipation : système anglais, système français ", Mots, 113, 9-36.

EISENBERG Peter (2017), « Wort des Jahres „Flüchtling“. Hier endet das Gendern », A. Meinunger \& A. Baumann (dir.), Die Teufelin steckt im Detail, Berlin : Kulturverlag Kadmos, 67-70.

ELMIGER Daniel (2008), La féminisation de la langue en français et en allemand, Paris : Honoré Champion.

FLEISCHER Wolfgang \& BARZ Irmhild (2012), Wortbildung der deutschen Gegenwartssprache, Berlin : De Gruyter.

HAUT CONSEIL À L'ÉGALITÉ (2016), Pour une communication publique sans stéréotype de sexe, Paris : La Documentation française, <http://bit.ly/2fejwZ7> (17 décembre 2020).

HERMANN Steffen Kitty (2003), « Performing the Gap. Queere Gestalten und geschlechtliche Aneignung ", arranca!, 28, 22-26, <https://arranca.org/archive? path=\%2Fausgabe\%2F28\%2Fperforming-the-gap> (17 décembre 2020).

HORNSCHEIDT Lann (2012), Feministische w_orte: ein lern-, denk- und handlungsbuch zu sprache und diskriminierung, gender studies und feministischer linguistik, Francfort-sur-le-Main : Brandes \& Apsel. 
INALF (1999), Femme, j'écris ton nom..., Paris : La Documentation française.

LE CALLENNEC Sophie \& FRANçoIs Émilie (2017), Magellan et Galilée. Questionner le monde. CE2, Paris : Hatier.

MEINUNGER André (2017), « Ein Plädoyer für das Deutsche als geschlechtergerechte Sprache - ein paar provozierende Beobachtungen und Ausführungen », A. Meinunger \& A. Baumann (dir.), Die Teufelin steckt im Detail, Berlin : Kulturverlag Kadmos, 93-100.

PAVEAU Marie-Anne (2002), « La féminisation des noms de métiers : résistances sociales et solutions linguistiques », Le français aujourd'hui, 136, 121-218.

PITTNER Karin (1998), « Genus, Sexus und das Pronomen wer », R.-J. Pittner \& K. Pittner (dir.), Beiträge zu Sprache und Sprachen, Munich : Lincom Europa, 2, 153-162.

RieGel Martin, Pellat Jean-Christophe \& Rioul René (1994), Grammaire méthodique du français, Paris : PUF.

SCHAFRoth Elmar (2010), « Die Janusköpfigkeit der Political Correctness », Zeitschrift für KanadaStudien, 30(2), 101-124.

SCHNEIDER-Mizony Odile (2007), « Ausdruck und Anrede im Femininum », I. Behr, A. Larrory \& G. Samson (dir.), Der Ausdruck der Person im Deutschen, Tübingen : Stauffenburg Verlag, 111-125. STEFANOWITSCH Anatol (2018), Eine Frage der Moral: Warum wir politisch korrekte Sprache brauchen, Berlin : Duden.

Violi Patrizia (1987), « Les origines du genre grammatical », Langages, 85, 15-34.

YAGUELLO Marina (1998), «Y a-t-il un français politiquement correct? », P. Singy (dir.), Les femmes et la langue. L'insécurité linguistique en question, Lausanne : Delachaux et Niestlé, 177-194.

YAGUELlo Marina (2014), Les mots ont un sexe, Paris : Points.

\section{NOTES}

1. INA (2019), Temps de parole des femmes: 700000 heures de programmes analysées, en ligne sur $<$ https://institut.ina.fr/actualites/temps-de-parole-des-femmes> (dernière consultation pour tous les liens de cette contribution : 29 novembre 2019).

2. Voir UNESCO (1989), Guidelines on Non-Sexist Language - Pour un langage non sexiste, en ligne sur <https://unesdoc.unesco.org/ark:/48223/pf0000097348.locale=fr>.

3. REY Alain, «L'écriture inclusive est vouée à l'échec », Le Figaro, 23 novembre 2017.

4. «Die Bildung neuer femininer Formen von Berufsbezeichnungen und die Kampfansage an das generische Maskulinum haben im Frankreich der 80er und 90er Jahre des letzten Jahrhunderts regelrechte ideologische Grabenkämpfe nach sich gezogen. "

5. Dans son emploi générique, iel permet de désigner toute personne sans distinction de genre ; dans son emploi spécifique, il permet de désigner toute personne non binaire.

6. G ESETZE-IM-INTERNET (2018), Personenstandsgesetz (PStG), en ligne sur <www.gesetze-iminternet.de/pstg/BJNR012210007.html>.

7. La présentation qui suit met l'accent sur la visibilité des femmes dans la langue, d'une part parce que la réflexion sur la déconstruction de la binarité du genre n'en est pas au même stade en France et en Allemagne, d'autre part parce que le traitement d'une question aussi complexe dans une perspective contrastive mériterait une étude à part entière. 
8. "[...] die Vermeidung nicht (mehr) opportuner Bezeichnungen für - aus unterschiedlichsten Gründen- als prekär oder heikel betrachtete Begrifflichkeiten oder Sachverhalte.» (Schafroth, $2010: 101)$

9. « Es bleibt allerdings zu hoffen, und hier beschwöre ich noch einmal das hässliche Gesicht der Political Correctness herauf, dass es in dieser Frage zu keinen interdictions - gleich welcher Art - kommen möge. » 10. Chronique « Le fin mot de l'info » diffusée sur Europe 1 le 26 novembre 2017.

11. Voir la une du magazine Marianne, $n^{\circ} 1081$ (1-7 décembre 2017) : « Les nouveaux censeurs ».

12. A CADÉMIE FRANÇAISE (2017), Déclaration sur l'écriture dite "inclusive», en ligne sur $<w w w$.academie-francaise.fr/actualites/declaration-de-lacademie-francaise-sur-lecriture-diteinclusive>.

13. «Greife nie in ein System ein, dessen Komplexität du nicht übersiehst. » (Leiss citée par SchneiderMizony, $2007: 120)$

14. « Ab dem Augenblick, wo Studentin neben Student, Beamtin neben Beamter, Kundin neben Kunde stehen, verliert das generische Maskulinum seine Generizität und wird mit einer männlichen Personenbezeichnung assoziiert. "

15. Sur la distinction entre ces deux procédés, voir Elmiger (2008 : 72-77).

16. CENTRE JURIDIQUE FRANCO-ALLEMAND (2004), Grundgesetz / Loi fondamentale (5e édition synoptique bilingue), en ligne sur <www.cjfa.eu/REPOSITORY/EDCJFA_3.pdf>.

17. Voir le rapport adopté à une large majorité le 28 février 2019 : ACADÉMIE FRANÇAISE (2019), La féminisation des noms de métiers et de fonctions, en ligne sur <http://www.academie-francaise.fr/ [...]noms_de_metier_et_de_fonction.pdf>.

18. W IKIPEDIA (2019), Virginie Basselot, en ligne sur <https://fr.wikipedia.org/wiki/ Virginie_Basselot> (12 novembre 2019).

19. W IKIPEDIA (2019), Anne-Sophie Pic, en ligne sur <https://fr.wikipedia.org/wiki/AnneSophie_Pic> (8 octobre 2019).

20. W IKIPEDIA (2019), Stéphanie Le Quellec, en ligne sur <https://fr.wikipedia.org/wiki/ St\%C3\%A9phanie_Le_Quellec> (24 octobre 2019).

21. W IKIPEDIA (2019), Julia Komp, en ligne sur <https://de.wikipedia.org/wiki/Julia_Komp> (23 octobre 2019).

22. WIKIPEDIA (2019), Maike Menzel, en ligne sur <https://de.wikipedia.org/wiki/Maike_Menzel> (19 octobre 2019).

23. WIKIPEDIA (2019), Douce Steiner, en ligne sur : <https://de.wikipedia.org/wiki/Douce_Steiner> (26 septembre 2019).

24. «Das dominierende Movierungssuffix ist heute -in [...]. » (Fleischer \& Barz, 2012 : 236)

25. Voir ce portrait d'Angela Merkel diffusé le 10 octobre 2005 sur France 3 (timecode 02:10): "Pour séduire les Allemands, madame la chancelière devra maintenant imposer ses idées libérales [...]. » INA (2005), Portrait Angela Merkel, en ligne sur <www.ina.fr/video/2945018001017>.

26. "Die Bezeichnung Friseuse wird nur noch in der Umgangssprache gebraucht; die offizielle Berufsbezeichnung lautet Friseurin. » (Duden online) NB : Le dictionnaire Wahrig de 1994 considère encore Friseurin comme un austriacisme.

27. DWDS (2019), Friseurin, en ligne sur <https://www.dwds.de/wb/Friseurin> et <https:// www.dwds.de/wb/Friseuse>.

28. «Die korrekte weibliche Berufsbezeichnung lautet Masseurin. Die Form Masseuse wird dagegen überwiegend in der Bedeutung Prostituierte gebraucht. » (Duden online)

29. L'adjonction du suffixe -in est attestée, mais pas (encore) considérée comme correcte : *die Flüchlingin (voir Fleischer \& Barz, 2012 : 237).

30. ATILF (1994), Trésor de la langue française informatisé, en ligne sur <http://www.atilf.fr/tlfi>. NB : il est significatif que la version en ligne du TLF, dont la rédaction est achevée depuis 1994 et qui n'est pas mis à jour, considère épicène comme un terme rare. 
31. Le féminin mairesse est anciennement attesté (voir INaLF, 1999 : 22), mais il a été victime de la dépréciation du suffixe -esse (comme doctoresse, poétesse, etc.).

32. Chevalier, Constantin De Chanay et Gardelle (2017:27) indiquent qu'au Québec, le double genre de membre est passé dans l'usage.

33. Elmiger ne considère comme épicènes que les noms de personnes à genre variable (2008: 67-68), ceux à genre fixe étant qualifiés par lui de noms génériques (2008: 83).

34. UNIVERSITÄT BREMEN (2014), Orientierungshilfe für eine gendergerechte Sprache an den Hochschulen im Land Bremen, en ligne sur <www.uni-bremen.de/fileadmin/user_upload/sites/zentralefrauenbeauftragte/UfHuql-OrientierungshilfeFuer[...]>.

35. " [...] wir haben im Deutschen sehr wohl schon lange und vollkommen unentdeckt ein generisches Femininum.»

36. «[...] im Plural [existiert] eine weibliche Vorherrschaft - im Singular eine männliche - es ist ausgeglichen. »

37. «Das angeblich generische Maskulinum wird überschattet von seiner sexusreferierenden Bedeutung und ist bei Stellen, in denen eine Frau als solche identifiziert werden sollte, immer schlechter möglich. » 38. «Ces jours et ces nuits entières » (Racine, 1691), cité dans le Guide du Haut Conseil à l'Égalité (2016 : 25). Voir aussi : VIENNOT Éliane (2018), L'accord de proximité, mode d'emploi, en ligne sur $<$ www.elianeviennot.fr/Langue-proxi.html>.

39. Par exemple la charte de l'université de Cologne : ÜberzeuGENDERe Sprache - Leitfaden für eine geschlechtersensible und inklusive Sprache (2014:19), en ligne sur <https://gedim.uni-koeln.de/ sites/genderqm/user_upload/Leitfaden[...].pdf>.

40. Exemple emprunté à Bußmann et Hellinger (2003: 165).

41. GRAMmis (2016), Das generalisierende Personalpronomen man, en ligne sur <https://grammis.idsmannheim.de/kontrastive-grammatik/3971>.

42. Comme la charte de l'université de Cologne précédemment citée (2014: 19).

43. CENTRE JURIDIQUE FRANCO-ALLEMAND (2004), Grundgesetz / Loi fondamentale (5 ${ }^{e}$ édition synoptique bilingue), en ligne sur <www.cjfa.eu/REPOSITORY/EDCJFA_3.pdf>.

44. S TUMMER Gabi (2012), "Geschlechtergerechtes Grundgesetz », en ligne sur GeschlechtergerechtesGG :

$<$ https://sites.google.com/site/geschlechtergerechtesgg/ geschlechtergerechtes-gg>.

45. U NIVERSITÄT LEIPZIG (2013), Grundordnung der Universität Leipzig, en ligne sur <www.unileipzig.de/fileadmin/ul/Dokumente/Grundordnung_UL_130806.pdf>.

46. «In dieser Ordnung gelten grammatisch feminine Personenbezeichnungen gleichermaßen für Personen männlichen und weiblichen Geschlechts. »

47. "Stelle andere sprachlich nicht so dar, wie du nicht wollen würdest, dass man dich an ihrer Stelle darstelle. » (Stefanowitsch, $2018: 24$ )

48. " Auch wenn einen das Mitgemeintsein nicht grundsätzlich stört, ist das eine leicht desorientierende und deshalb sehr lehrreiche Erfahrung [...]. » (Stefanowitsch, 2018 : 44)

\section{RÉSUMÉS}

Cette contribution porte sur les modalités de mise en œuvre du langage inclusif en français et en allemand. Après une synthèse des objections que cette atteinte politiquement correcte à la règle du masculin générique suscite dans les deux aires linguistiques, l'accent est mis sur les faits de 
langue concernés et sur les solutions proposées en français et en allemand pour surmonter cet androcentrisme hérité de la tradition patriarcale. L'allemand possède l'avantage de n'accorder ni ses participes ni ses adjectifs attributs, mais certains pronoms difficiles à éviter dans la communication courante, tels que l'indéfini man (on) et le relatif wer (qui), contribuent à maintenir le caractère générique du masculin en langue et en discours. En français, si la recherche de l'équité en genre se heurte notamment à l'accord des adjectifs et participes, les nombreux noms de personne à finale en - $e$ facilitent grandement le processus d'épicénisation. $\mathrm{Au}$ terme de cette étude comparée, il apparaît que la langue la plus apte au rééquilibrage des genres n'est peut-être pas celle qu'on croit.

This article discusses the ways in which inclusive language is employed in French and German. First, a synthesis is offered of the objections that this politically correct violation of the generic masculine rule raises in both linguistic areas. The article then addresses the relevant linguistic facts and the solutions proposed in French and German which obviate this androcentric linguistic structure inherited from a long patriarchal tradition. While German has the advantage that its participles and predicative adjectives are invariable, some hard-to-avoid pronouns, such as the indefinite man (one) and the relative wer (who), work to perpetuate the generic masculine in language and discourse. French agreement of participles and adjectives likewise inhibit attempts to achieve gender equality in the language, yet the numerous personal nouns ending with $-e$ make the creation of gender neutral terms much easier. This comparative study concludes that the language most capable of equalizing gender relations is not the one that would be most expected.

Dieser Beitrag setzt sich mit der Umsetzung einer gendergerechten Sprache im Französischen und im Deutschen auseinander. Zuerst wird auf die Einwände eingegangen, die dieser politisch korrekte Verstoß gegen die Regel des generischen Maskulinums in beiden Sprachräumen hervorruft, dann richtet sich der Fokus auf die betreffenden sprachelemente und auf die in beiden Sprachen vorgeschlagenen Lösungen zur Überwindung eines aus der patriarchalischen Tradition übernommenen Androzentrismus. Das Deutsche besitzt zwar den Vorteil, dass Partizipien und prädikative Adjektive nicht kongruieren, dennoch tragen manche Pronomen - wie das indefinite man und das Relativpronomen wer- deren Gebrauch in der Alltagskommunikation schwer vermeidbar ist, dazu bei, den generischen Charakter des Maskulinums in Sprache und Diskurs aufrechtzuerhalten. Im Französischen, wo die Bemühungen um Gendergerechtigkeit u. a. durch die Kongruenz von Partizipien und Adjektiven erschwert werden, erleichtern andererseits die vielen Personennamen mit Endung auf $-e$ die Einführung von neuen genderneutralen Bezeichnungen erheblich. Am Ende dieser vergleichenden Studie stellt sich heraus, dass die Sprache, in der ein Gleichgewicht der Geschlechter am ehesten zu erreichen wäre, möglicherweise nicht diejenige ist, die frau sich denkt.

\section{INDEX}

Schlüsselwörter : politische Korrektheit, Gender, Sexismus, Feminisierung, generisches Maskulinum

Mots-clés : politiquement correct, genre, sexisme, féminisation, masculin générique

Keywords : political correctness, gender, sexism, feminization, generic masculine 


\section{AUTEUR}

\section{NATHALIE SCHNITZER}

Aix Marseille Univ., ÉCHANGES, Aix-en-Provence, France.

Nathalie Schnitzer est germaniste, maîtresse de conférences à l'Université d'Aix-Marseille, membre de l'équipe Échanges EA 4236. Ses recherches portent sur le genre en langue et en discours, la grammaire et la didactique de l'allemand, la traductologie.

nathalie.schnitzer@univ-amu.fr 